\title{
Numerical Simulation of Quasi-Static Compression Behavior of the Toe Cap Component for Safety Footwear
}

\author{
Sérgio L. Costa, João P. Mendonça, and Nuno Peixinho
}

\begin{abstract}
Geometrical changes can improve stiffness substantially. The project S3 - Safety Slim Shoe presents the potential to reduce the weight in safety toe cap components combining a new geometric redesign deeply associated to local stiffeners to realize the full potential of AHSS - Advanced High Strength Steels. The investigation aimed to examine the potential energy absorption capacity for a substantial thickness reduction of slim toe cap models. In this paper the normative quasi-static compression test in the context of the experimental validation of the two last and approved prototype models were focused. A non-linear FEA - Finite Element Analysis of the elasto-plastic deformation mode was performed, and several numerical parameters such as: hardening effects of extrapolated True-Stress-Strain material curves and simulation convergence conditions were carried out. Experimental results of the toe cap deformation behavior confront a weight saving range of over $40 \%$, compared with the original steel toe cap.
\end{abstract}

Index Terms-AHSS, FEA, local stiffening, toe cap.

\section{INTRODUCTION}

The safety footwear, in the context of the global evolution of footwear, has adopted creative and different orientations. Particularly, as an active element in the prevention of accidents fitted in PPE - Personal Protective Equipment, it is appropriate that its optimization solutions amplify the market targets, mainly combining ergonomic aspects, biological and mechanical features since its conception. The toe cap is the main component for its weight contribution, approx. $35 \%$ of total weight for each safety footwear model, and due normative context demands with high deformation resistance and impact loading [1]. Currently the toe cap protection takes a subdivided position on design selection and materials, with a clear definition of two distinctive conception trends: metallic and non-metallic models. If on one hand the metallic models, especially high carbon steels with Heat Treatment processes, emphasize the security concept by the implicit mechanical strength performance, on the other hand, the weight of these solutions is a disadvantage compared to the metal-free solutions, and relegates the first one for outdated and heavier footwear concept. The non-metallic solutions with higher trend effects in the global market call on low density materials such as: reinforced Polyester composites with glass fiber, HDPE - High Strength Polyethylene, and several advanced developments in the optimization of energy

Manuscript received September 25, 2013; revised January 2, 2014

The authors are with the Centre for Mechanical and Materials Technologies - CT2M, Department of Mechanical Engineering, University of Minho, 4800 - 058, Guimarães, Portugal (e-mail: \{sergio.costa, jpmas, peixinho\}@dem.uminho.pt). absorption properties [2]-[9]. These preceding solutions are currently lighter than referenced steel toe caps, achieving approx. $40 \%$ of weight decrease [10]-[13]. Despite of these composite and hybrid material solutions, its main disadvantage is still committed to the mechanical strength performance, when compared to the steel toe caps, requiring a larger volume concept (to counterpoise higher rates of deformation) and in several ways that affects the conception of the main integrant parts. Both distinguished models and the ultimate S3 prototype are shown in Fig. 1.
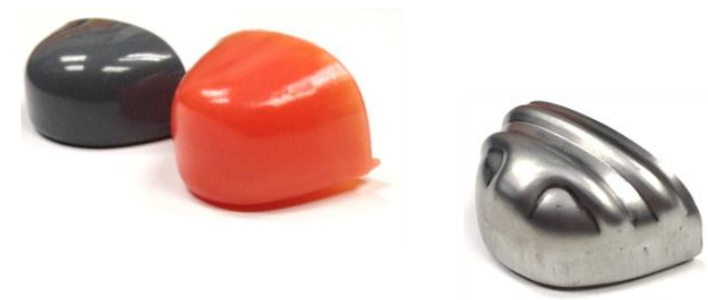

Fig. 1. Toe cap models with equivalent size: original steel toe cap, composite toe cap and S3 Final model prototype.

The research Project S3 - Safety Slim Shoe aims to complement the properties of the two referenced solutions, promoting the industrial development of a brand new ultimate toe cap solution. A substantial reduction of weight due the thickness optimization, and lower volume rates compared with the polymeric solutions are goals for the framework.

In this paper, the contribution of the parameters under study for the energy absorption optimization was focused on the elasto-plastic behavior to quasi-static compression tests. In this context, the current progress of the project S3 brought here the two latest and approved toe cap prototype models, the S3 Stage II model and the Final model (with specific corrections for the complementary dynamic normative test), with thickness combinations of 1.2 and $1.1 \mathrm{~mm}$. This study presents a FEA to compare among the performance achieved with these models and the original reference with $1.9 \mathrm{~mm}$ thickness, concerning static stiffness values duly calculated in plastic domain. The opportunity to evaluate some critical parameters related to the numerical simulation of a particular case study with complex geometric design was also covered. Experimental results were briefly attempt.

\section{QuASI-STATIC COMPRESSION TEST}

The European Standard requires the high performance level of the toe cap components before being placed into the footwear product in the normative context of EN 12568:2010 [1]. Fig. 2a) represents the normative test used in this study with the schematic configurations and the relative position of the test specimen. The deformation mode of a steel alloy, 
resulting from the normative quasi-static compression test, reveals a standard curve shown in Fig. 2b).
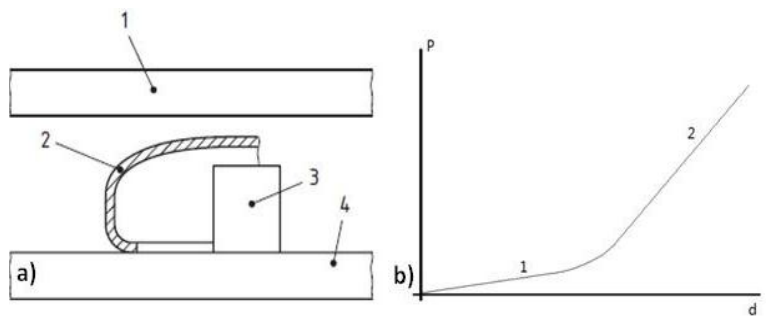

Fig. 2. Normative context: a) Apparatus for compression test. Key: (1) Upper platen, (2) Toe cap, (3) Modelling clay cylinder, (4) Lower platen [1]; b) Load vs. displacement typical curve for steel toe cap under static compression test [1].

The test method consists in the compression of the test specimen by a controlled computed dynamometer to a load of either $(15 \pm 0.1) \mathrm{kN}$ for toe caps to be used for safety footwear class, by moving the upper platen at a speed of $(5 \pm$ 2) $\mathrm{mm} / \mathrm{min}[1]$. When toe caps are tested in accordance with the method described, the final clearance under the toe cap shall not be less than the appropriate value for the size of the model [1].

The straight line (1) shown in Fig. 2b) matches to the elastic deformation domain of the material, and can be defined by a stiffness constant, equal to the slope of the respective line. The straight line (2) corresponds to the plastic deformation, after forming the 1st plastic hinge. This line can also be defined by a constant stiffness equivalent to its slope. This means that the formation of the second and last plastic hinge occurs to the higher load value when the test ends at 15 $\mathrm{kN}$. The large reduction of thickness by upgrading the toe cap model and consequent static stiffness loss, an important phenomenon into the continuous loading application, may lead to intermediate hinges occurring in the elasto-plastic deformation and cause a collapse mode. In this case, the performance of the deformation resistance is compromised.

\section{DESIGN PARAMETERS}

\section{A. Local Stiffening}

From a design point of view, the high stresses and low thickness put emphasis on finding a solution that uses the full potential of the material. Design challenges for a new toe cap model have included some complex phenomena's such as structural stiffness and, in this case, the quasi-static deformation resistance. Thus, local buckling may occur when reducing thickness during an upgrading process. This is initially an elastic instability phenomenon, where local buckles form at stresses below the plastic limit and in doing so, do not use the full potential of the material (further, it can be observed in the quasi-static compression test at the first plastic hinge formation). Introducing local stiffeners to reduce the increased slenderness and improve the constraint of the edges helped to converge the potential of AHSS solution. Fig. 3 shows the stiffeners of the S3 intermediate model considered in this study. Since mild steel and high strength steel have close values of Young's modulus, the stiffness of a single toe cap component is lost in an upgrading situation where the thickness is intentionally reduced if all other parameters are kept constant. One way to fulfil the stiffness demands on a design is to change the outer geometry [14], [15].

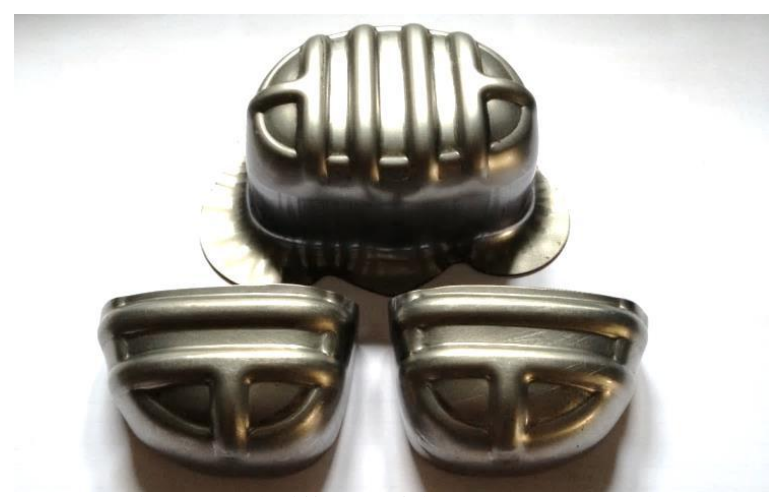

Fig. 3. Local stiffeners of the S3 Stage II model.

The high strength steels used in project S3 have shown a combination of special mechanical properties such as high tensile strength, high work hardening rate at early stages of uniform plastic deformation and good formability, combining a high energy absorption potential for dynamic and quasi-static loading [16], [17]. For the S3 toe cap development, the optimization of the energy absorption was properly related to the evolution of each deformation model, concerned with the different behaviour responses for quasi-static compression and impact-crash tests. In this study, the first one was exclusively carried out and three different AHSS steels combining a thickness range between $1 \mathrm{~mm}$ and $1.2 \mathrm{~mm}$ were performed. Fig. 4 represents the True-Stress-Strain extrapolated curves properly applied in the numerical simulation.

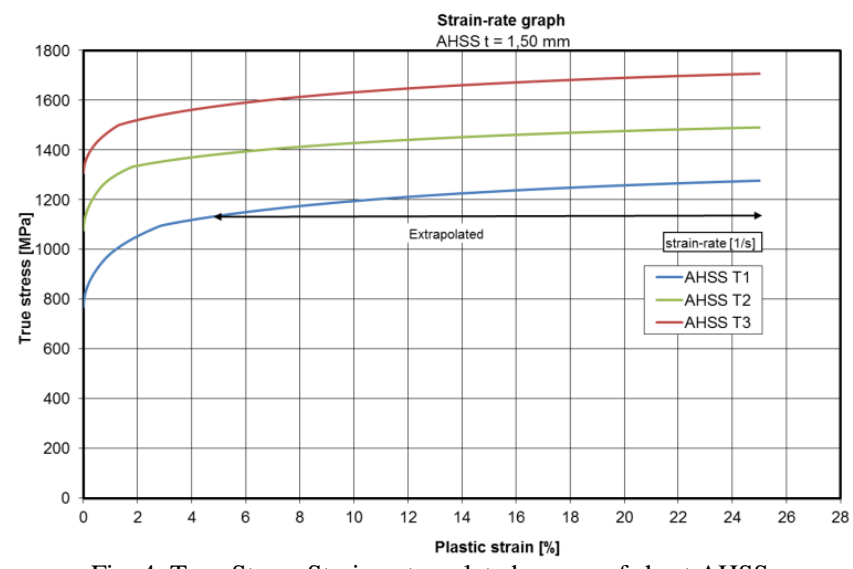

Fig. 4. True-Stress-Strain extrapolated curves of sheet AHSS.

\section{B. Reverse Engineering and Prototypes}

The dimensional control associated with the toe cap is determined taking into account the process of manufacturing the safety shoe and the type of mechanisms used to mount the shoe. It had to be ensured that the CAD model is obtained geometrically according to the physical model, especially in the surface assembly points. The basic conceptual model for the computational development of the original steel toe cap, and the redesign process of new geometric models has experimented a reverse engineering approach detailed in Fig. 5

The set of point clouds from the surface measurement was collected by a scanning laser technique. In the next step, the information provided by data acquisition was preprocessed 
with an auxiliary advanced algorithm in Matlab® to improve native geometrical entities for the basis surface construction. Then, the optimization has passed through the re-design process with the development of several geometrical changes. Local stiffeners and other features were implemented into powerful surface modeling tools in CATIA ${ }^{\circledR}$ [18]. Fig. 6 shows the original steel toe cap and the S3 Final model prototype taken in this study.

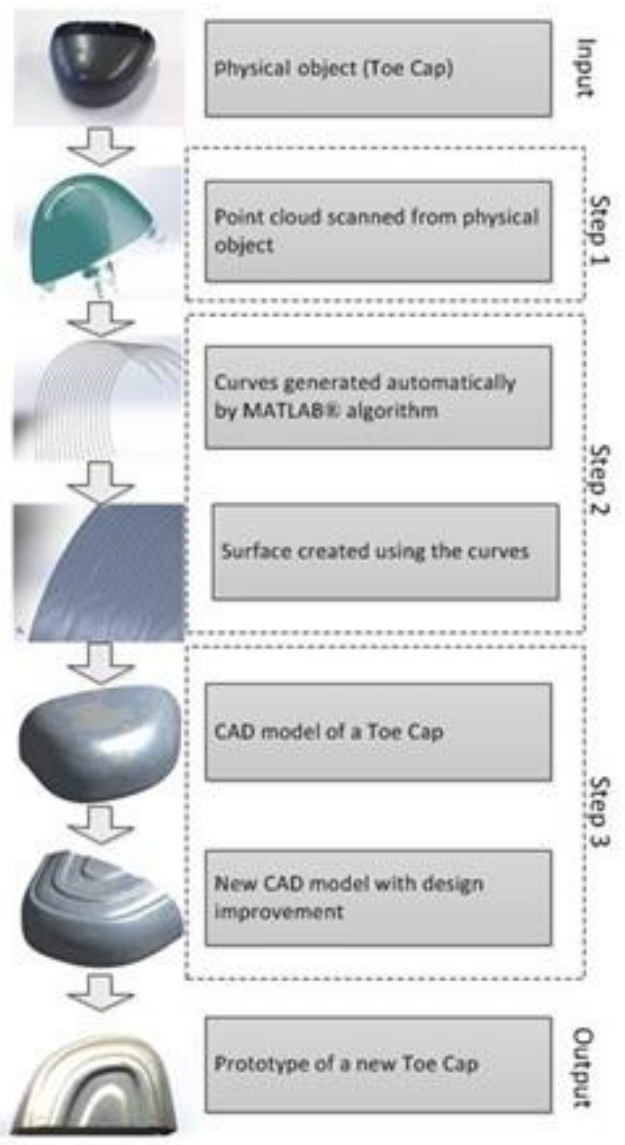

Fig. 5. Reverse engineering methodology for Project S3 [18].
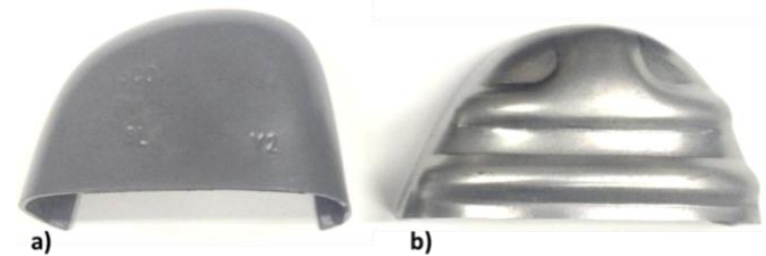

b)

Fig. 6. Toe cap models: a) original steel toe cap and b) S3 Final prototype.

\section{NUMERICAL SIMULATION}

The FEA - Finite Element Analysis of toe cap models on non-linear study made for static load conditions was performed with an implicit FE code, ANSYS (Workbench). Static compression tests were simulated for each respective combination of study parameters: thickness, material and geometric model. Fig. 7 shows the finite element environment with the upper platen of the load cell test in accordance with the normative method previously described. The boundary conditions were defined with axial loading application on the upper platen, and the fixed support in the tab region was reproduced. An average load value of $4 \mathrm{kN}$ to cover the elastic domain behavior of the toe cap models response was applied. The force application body was considered rigid and without analysis settings in order to transfer the entire energy recording to the toe cap component. Remote displacements of the upper platen and other dependent surfaces were added with the purpose of constraining the load body, in rotation and other directional components, to promote a guided displacement evolution. The level of plastic deformation had increased following the first plastic hinge formation and brought some simulation challenges. Higher deformation rates leads to force convergence errors in the increment model of the deformation analysis and consequently the interruption of the numerical progress [19].

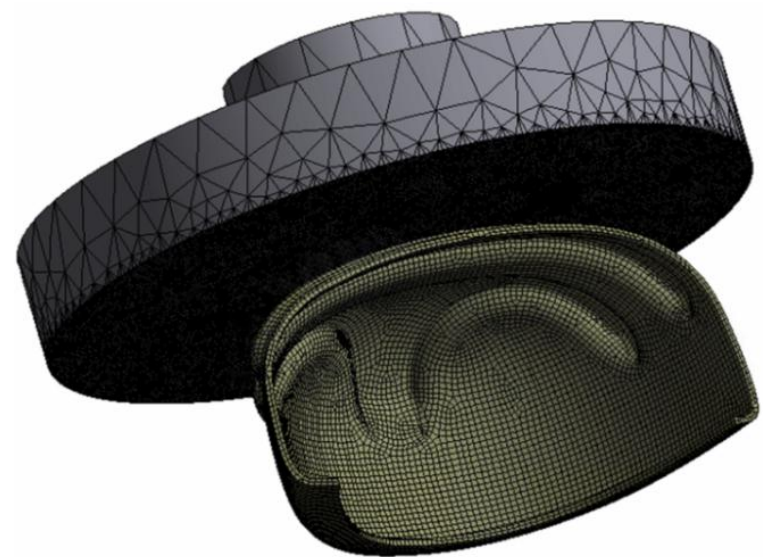

Fig. 7. FEM of the quasi-static analysis for the S3 Final model. ANSYS ®.

A prior attention target in the numerical simulation was the body's connection with the contact part, between the virtual toe cap model and the pressure plate above. The area of contact, ideally converged to a contact point border between the bodies according to the normative test method, brought here some simulation improvement needs. In this context, small contact extensions were attempt and the friction coefficient was also an influent parameter. For a range value between 0.32 and 0.74 the evaluation was 0.54 [19]. The mesh element generation and the type of contact were applied differentially and have been under convergence studies. Nonlinear solution is sensitive to mesh by the number of elements and the respective method (shape of elements). Mesh quality has shown not only a matter of accuracy and runtime for the solution but also a matter of success or failure. In general, hexahedra are more efficient than tetrahedra elements [19]. In this case, simulation models had considered hexahedra as many as possible, by the geometric complexity, performing the Hex-Dominant method. Fig. 8 shows the comparison of the mesh parameters. As expected, the Higher-Order Hexahedra elements (element midside nodes kept) performed better results for a fine mesh resulting on higher values of directional deformation. Thus, this method and an element size control stabilized between 2 and $2.3 \mathrm{~mm}$ were selected to the main bodies (56.149 nodes). Nevertheless, the inevitable progress of the complex plastic deformation under harder and continuous loading conditions with micro cracks initiation and fracture phenomenon promoted at the end of the material behavior has been succeed up to $10 \mathrm{kN}$. Although the interruption of the implicit numerical simulation ocurred, it was important to perform the evaluative static deformation response to confront different combinations of geometric models and AHSS materials 
converging on the best design changes. The plastic hinges associated to the static stiffness and deformation models were rated.

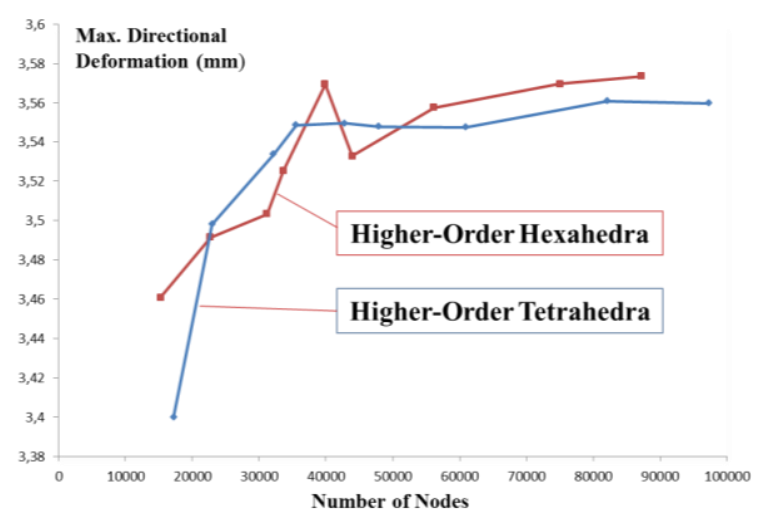

Fig. 8. Mesh convergence analysis for the S3 Stage II model performance.

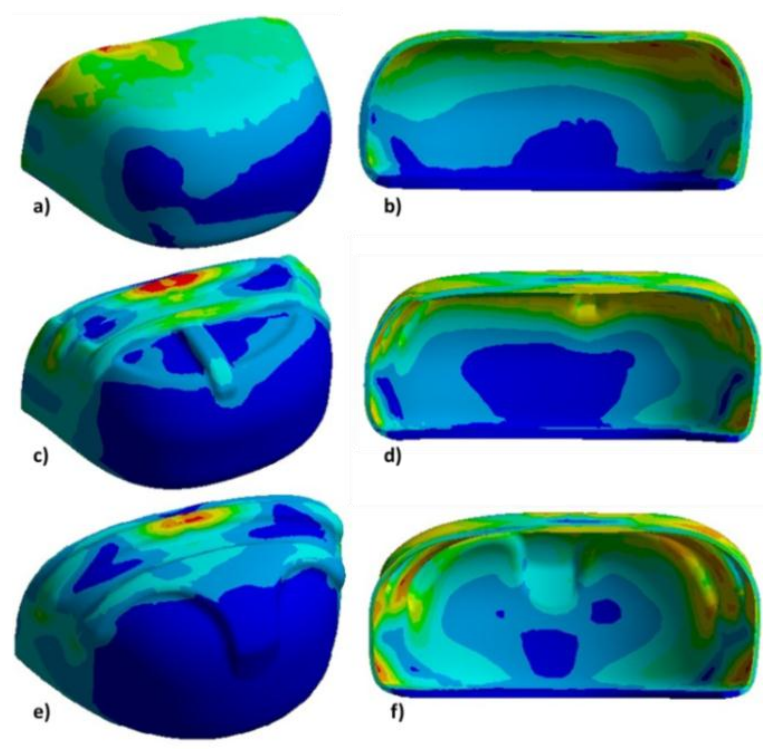

Fig. 9. Stress distributions - elastic deformation of AHSS T2 $1.2 \mathrm{~mm}$ thick models from FEA for $2500 \mathrm{~N}$ : a) original steel toe cap, c) S3 Stage II and e) S3 Final. Stress distributions - plastic deformation for $10 \mathrm{kN}$ : b) original steel toe cap, d) S3 Stage II and f) S3 Final. ANSYS® Environment.

The numerical simulation program was applied to the new geometric models with the three abovementioned AHSS and conjugating in thicknesses between $1.0 \mathrm{~mm}$ and $1.2 \mathrm{~mm}$. Isotropic elasticity and multi-linear isotropic hardening parameters were defined. Potential solution models were compared with the original steel toe cap model. Fig. 9 shows representative results of the quasi-static FEA analysis for the new S3 models with the combination of $1.2 \mathrm{~mm}$ thickness and AHSS T2 material, and the original steel toe cap model of $1.9 \mathrm{~mm}$ thickness for the C65 Heat Treated alloy. Compressive loading conditions of $10 \mathrm{kN}$ were performed for the S3 Stage II, S3 Final and the original steel toe cap model. Stress concentrations occurred in the contact region with the upper platen point inducing higher force moments on the top toe cap surface, which affected much the overall deflection and damage of toe cap. For these load values it can be observed a slightly different mode of deformation for the proposed models. The S3 Stage II and the Final geometries provided more transversal deformation characteristics instead of the original steel toe cap that promoted a larger deflection. The results of the static deformation behavior are plotted in Fig. 10a to compare performances of the S3 Stage II with the original model, and the comparison between S3 models in Fig 10b).

Table I brings together the first plastic hinge, where the plastic deformation begun after springback effects that is much associated to the elastic deformation field, and the last plastic hinge that the FEA analysis had performed with the reaction force and the resulting displacement values for each model. The equivalent stiffness constants of the plastic deformation behavior were calculated for these points. Consequently, the performance of the energy absorption was analyzed.

The numerical simulation was not completed up to $15 \mathrm{kN}$ as demanded by the normative and experimental context for any toe cap model, because models required to higher strain rates have revealed earlier problems associated with the interruption of the FEA as explained above.
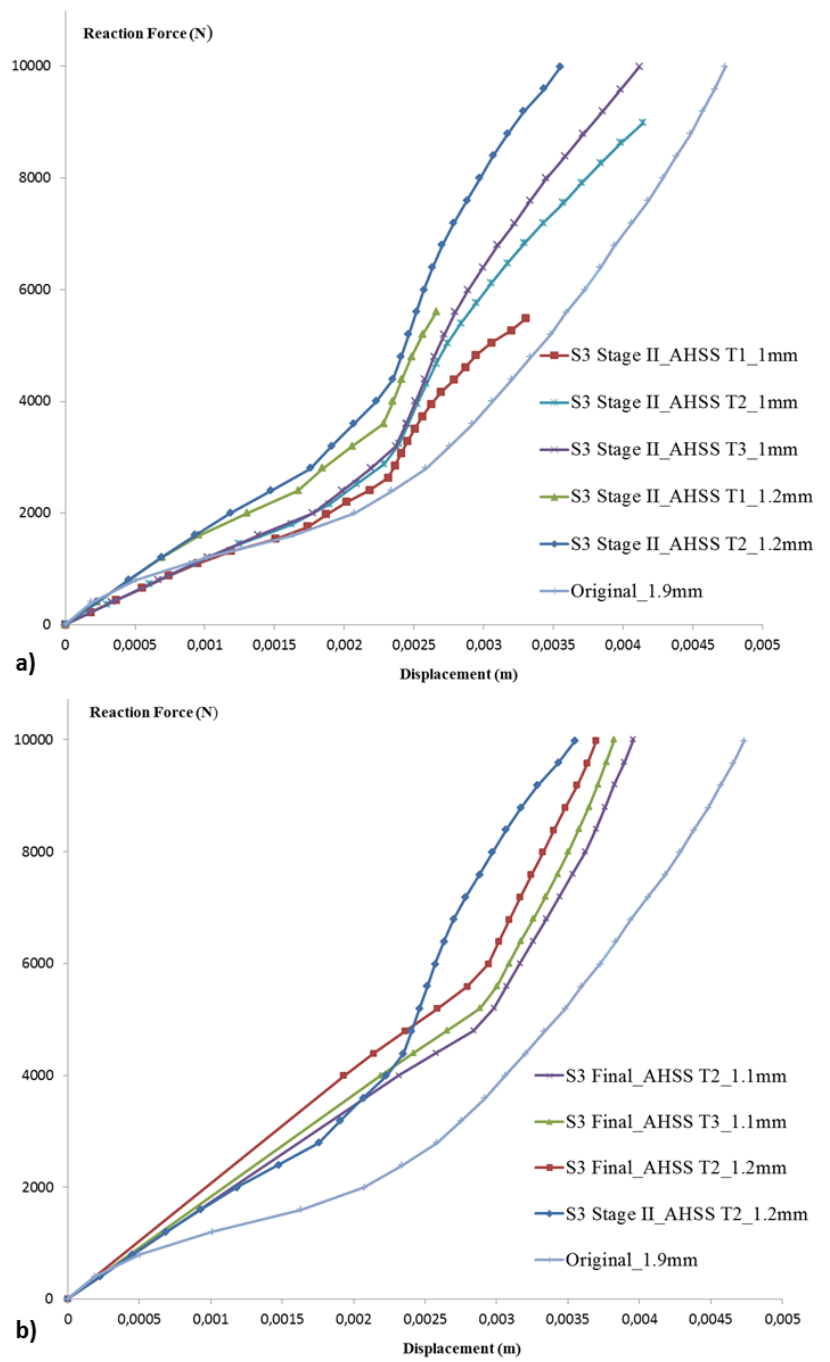

Fig. 10. Numerical results of quasi-static compression tests: a) comparison of S3 Stage II models and the original steel toe cap, b) comparison between the two S3 models and the original steel toe cap.

From the global numerical results it was found that the S3 Stage II model performed the higher capacity for energy absorption in the plastic deformation range, reflecting to the higher value of stiffness constant (Table I). The effect of the structural shoulder on the toe cap side walls, due to the increased extension of the lateral ribs, contributes to deformation mode benefits. Increasing strain rate effects, 
with thickness reduction to $1 \mathrm{~mm}$ and the first AHSS grade, has increased problems related to the phenomena of stiffness loss and the breaking of static curves was occurred. Numerical results for the plastic behavior confirmed that the parameters under study have relevant and different contribution. Geometric and thickness factors were most critical in the performance of energy absorption than the selection of AHSS (shown in Fig. 10b), which means that design changes improve stiffness substantially as aforementioned, and that has been fundamental to potentiate the AHSS contribution on weight savings. The combined S3 Stage II AHSS T2 $1.2 \mathrm{~mm}$ model achieved the better performance due to the formation of the last plastic hinge with the smaller displacement value and, consequently, the higher line slope and stiffness value.

In comparison with the S3 Final model, with the same AHSS and thickness values, it was found a slightly increase of the plastic stiffness constant. It is pertinent at this point to note that the S3 Final model is a close and corrective evolution to the previous S3 Stage II model in the context of another complement test, with a different demanding behavioral mode. Thus, the specific geometric features of the S3 Final surface, more spherical, had not converged in the present test as a top priority concern. Nonetheless, the S3 Final model had announced the higher value for the elastic stiffness, as shown in Table I, and has revealed a lower sensitivity to the AHSS selection and thickness decrease.

TABLE I: NUMERICAL RESULTS OF QUASI-STATIC TESTS

\begin{tabular}{|c|c|c|c|c|c|c|}
\hline \multirow[b]{2}{*}{ Toe cap model } & \multicolumn{2}{|c|}{$1^{\mathrm{a}}$ Plastic hinge } & \multicolumn{2}{|c|}{ Last plastic hinge } & \multicolumn{2}{|c|}{$\begin{array}{l}\text { Stiffness c. } \\
(\mathrm{N} / \mathrm{mm})\end{array}$} \\
\hline & $\begin{array}{l}\text { Force } \\
\text { reaction } \\
(\mathrm{N})\end{array}$ & $\begin{array}{l}\text { displ. } \\
(\mathrm{mm})\end{array}$ & $\begin{array}{l}\text { Force } \\
\text { reaction } \\
(\mathrm{N})\end{array}$ & $\begin{array}{l}\text { displ. } \\
(\mathrm{mm})\end{array}$ & Elastic & Plast \\
\hline S3 Stage II_AHSS T1_1mm & 1594.2 & 1.61 & 5486.1 & 3.30 & 990 & 2303 \\
\hline S3 Stage II_AHSS T2_1mm & 1913.5 & 1.76 & 8981.6 & 4.14 & 1087 & 2970 \\
\hline S3 Stage II_AHSS T3_1mm & 2073.3 & 1.82 & 9981.6 & 4.17 & 1139 & 3365 \\
\hline S3 Stage II_AHSS T1_1.2mm & 2234.2 & 1.57 & 9980.6 & 4.51 & 1423 & 2635 \\
\hline S3 Stage II_AHSS T2_1.2mm & 2713.6 & 1.73 & 9984.7 & 3.50 & 1569 & 4108 \\
\hline S3 Final_AHSS T2_1.1mm & 3999.9 & 2.31 & 9999.8 & 3.96 & 1732 & 3636 \\
\hline S3 Final_AHSS T3_1.1mm & 3999.8 & 2.19 & 9999.9 & 3.82 & 1826 & 3681 \\
\hline S3 Final_AHSS T2_1.2mm & 4788.9 & 2.36 & 9982.1 & 3.70 & 2029 & 3876 \\
\hline Original $1.9 \mathrm{~mm}$ & 2551.5 & 2.47 & 9982.6 & 4.73 & 1033 & 3288 \\
\hline
\end{tabular}

In this comparison between the conceptual S3 models, for the same AHSS and thickness, the S3 Stage II shows a stiffness increase of 3876 to $4108(\mathrm{~N} / \mathrm{mm})$, in order of $1.06 \mathrm{xs}$ in plastic behavior. On the other hand, confronting these close values with the original toe cap model $(1,25 \mathrm{x}$ lower even with $1.9 \mathrm{~mm}$ thickness) proves the optimization of the energy absorption and the design improvement of the ultimate S3 models. Thus, numerical results accounted up $10 \mathrm{kN}$ show that S3 Stage II and Final model for any material, thickness range and both level of material deformation have performed better results than the original $1.9 \mathrm{~mm}$ model.

The deformation curve of the original model presented a differential curvature characterized by a hardest identification of the formation of the first plastic hinge. Herewith the transition between the elasto-plastic domains revealed more linear.

\section{EXPERIMENTAL TESTS}

\section{A. Prototype Results}

The Quasi-static compression test in the normative experimental context was performed for the previous toe cap prototypes.

The experimental results are plotted in Fig. 11, and experimental stiffness constant values are presented in Table II. The S3 Stage II model has confirmed the predictions of the numerical simulation results, as the geometric model with higher energy absorption in the plastic behaviour. S3 Stage II AHSS T2 with $1.2 \mathrm{~mm}$ thickness prototype performed better experimental results leading to the last plastic hinge with the smaller displacement value. Experimental tests also validated the assumption of the different effect of study parameters. Geometry and thickness reduction factors had more sensitivity in the static compression test than the selection of the AHSS material type. Several plastic deformation effects after the loading condition of $10 \mathrm{kN}$ to the first AHSS and thickness reduction, has perceived for the new geometric prototypes with failure modes and the quasi-static test curve of the original model has shown different curvature resistance characteristics.

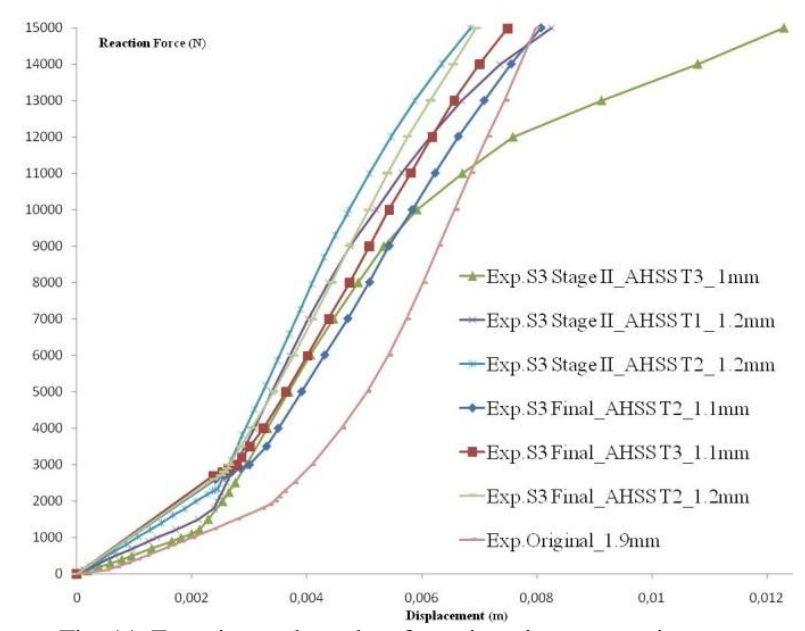

Fig. 11. Experimental results of quasi-static compression tests.

TABLE II: EXPERIMENTAL RESULTS OF QUASI-STATIC TESTS

\begin{tabular}{l|cccc|cc}
\hline \multicolumn{1}{c|}{ Toe cap model } & \multicolumn{2}{|c|}{$1^{\text {a }}$ Plastic hinge } & \multicolumn{2}{c|}{ Plastic hinge (equal } \\
for numerical hinge) & \multicolumn{2}{|c|}{$\begin{array}{c}\text { Stiffness const. } \\
\text { (N/mm) }\end{array}$} \\
& $\begin{array}{c}\text { Force } \\
\text { reaction } \\
(\mathrm{N})\end{array}$ & $\begin{array}{c}\text { displ. } \\
(\mathrm{mm})\end{array}$ & $\begin{array}{c}\text { Force } \\
\text { reaction } \\
(\mathrm{N})\end{array}$ & $\begin{array}{c}\text { displ. } \\
(\mathrm{mm})\end{array}$ & Elastic & Plastic \\
\hline S3 Stage II_AHSS T1_1mm & 1201.9 & 2.60 & 5501.9 & 4.64 & 462 & 2108 \\
\hline S3 Stage II_AHSS T2_1mm & 1101.3 & 2.11 & 9011.8 & 5.36 & 522 & 2434 \\
\hline S3 Stage II_AHSS T3_1mm & 1225.8 & 2.10 & 10002.9 & 5.92 & 584 & 2298 \\
S3 Stage II_AHSS T1_1.2mm & 1810.0 & 2.39 & 10007.8 & 5.21 & 757 & 2907 \\
S3 Stage II_AHSS T2_1.2mm & 2351.1 & 2.46 & 9993.4 & 4.74 & 956 & 3352 \\
\hline S3 Final_AHSS T2_1.1mm & 2901.8 & 2.86 & 10003.0 & 5.84 & 1015 & 2383 \\
S3 Final_AHSS T3_1.1mm & 3011.9 & 2.80 & 10007.8 & 5.44 & 1076 & 2650 \\
S3 Final_AHSS T2_1.2mm & 3011.9 & 2.66 & 10007.8 & 5.08 & 1132 & 2891 \\
\hline Original 1.9mm & 1900.9 & 3.30 & 10007.8 & 6.57 & 576 & 2479 \\
\hline
\end{tabular}

Hence, the influence of the redrawn geometry into energy absorption capacity has been proven, with stronger deformation resistance in early stages but also the challenge of balancing the stiffness loss with the substantial thickness reduction.

The S3 Stage II and S3 Final AHSS T2 1.2 mm prototypes confirmed similar performance values as can be seen in Fig. 11. The analysis of the respective curves from the quasi-static test to the thickness value of $1.2 \mathrm{~mm}$ shows a high resistance to static deformation and the S3 models have outperformed the original steel toe cap $1.9 \mathrm{~mm}$ model. The stiffness values of the Table III materialize the test results. The S3 Final 
model reaches the higher stiffness constant of the elastic deformation and an increase of roughly $50 \%$ compared to the original model. In this context, the energy absorption capacity revealed to the sringback effect retards the formation of the first plastic hinge and turns out to impair the calculation of the model performance in the plastic domain, compared with the S3 Stage II model. The maximum optimization considering better results than the original steel toe cap model with $1.9 \mathrm{~mm}$ of thickness value was performed by the S3 Final AHSS T3 $1.1 \mathrm{~mm}$ prototype.

\section{B. Accuracy Analysis}

In qualitative terms, the comparison of numerical and experimental results is significantly improved. Plastic hinges and the calculated values for stiffness constants of experimental test results are shown above in Table II and were equivalent for the same limit load values achieved in numerical simulation. Picking up the previous numerical example, the experimental results announce for S3 stage II and S3 Final geometric model assessment for the same AHSS material and thickness of $1.2 \mathrm{~mm}$, the stiffness constant values of 3352 and $2891(\mathrm{~N} / \mathrm{mm})$, respectively and equivalent to a ratio of approx. $1.15 \mathrm{xs}$ order $(1.06 \mathrm{xs}$ for numerical results).It was found for the same S3 Stage II AHSS T2 model with a thickness evaluation between 1 and $1.2 \mathrm{~mm}$, the experimental stiffness constant values of 2434 and 3352 $\mathrm{N} / \mathrm{mm}$, respectively and equivalent to an increase of approx. $1.37 \mathrm{xs}$ order (2970 and $4108(\mathrm{~N} / \mathrm{mm})$, equivalent to $1.38 \mathrm{xs}$ for numerical results). Hence, the accuracy of the numerical thickness evaluation of the S3 Stage II AHSS T2 model was approx. $98 \%$ and the accuracy of the numerical study of the geometric model evolution with the two last S3 models had reached approx. 92\%.

Quantitatively, the difference between experimental and numerical results is exemplified in Fig. 12 for two toe cap models: the original steel toe cap model and the S3 Stage II AHSS T2 $1.2 \mathrm{~mm}$ model. Related causes such as: stiffness loss, thinning effects associated with forming processes for advanced steels, differential thickness values of toe cap components, and issues associated with the numerical simulation, such as the definition of boundary conditions, convergence criterion parameters of the FE code, among others, are justified. The influence on the accuracy of numerical simulation of the prototype thickness decrease, considering a range of thinning effects of around 5 to $10 \%$, was exemplified and performed for the same S3 Stage II $1.2 \mathrm{~mm}$ model, considering a fact-finding $1.1 \mathrm{~mm}$ thickness model (Fig. 12). The results have converged on the accuracy of previous experimental values.

In this context, another hypothetical factor has been studied. The clamping position set and the support system of the test models have been reconsidered for the numerical simulation of the impact test. The specific deformation mode considering the deflection phenomenon of the toe cap side walls from the test evolution, had led here in some cases, and not so provided in normative conditions (although it did not predict the thickness optimization carried out), to attachment loss between the lower plate and the toe cap tab region. Slip effects were commonly verified in this boundary condition. A hybrid fixed support was further developed for the numerical simulation model by an evolutionary condition between fixed support, to initial time steps, and a final contact provided by a dynamic friction coefficient starting from frictionless to 0.32 [19]. Fig. 13 compares the sliding behavior of the side walls from the S3 final AHSS T2 $1.2 \mathrm{~mm}$ model, substantially changing the deformation mode, when considering the new support and the normative boundary conditions previously considered.

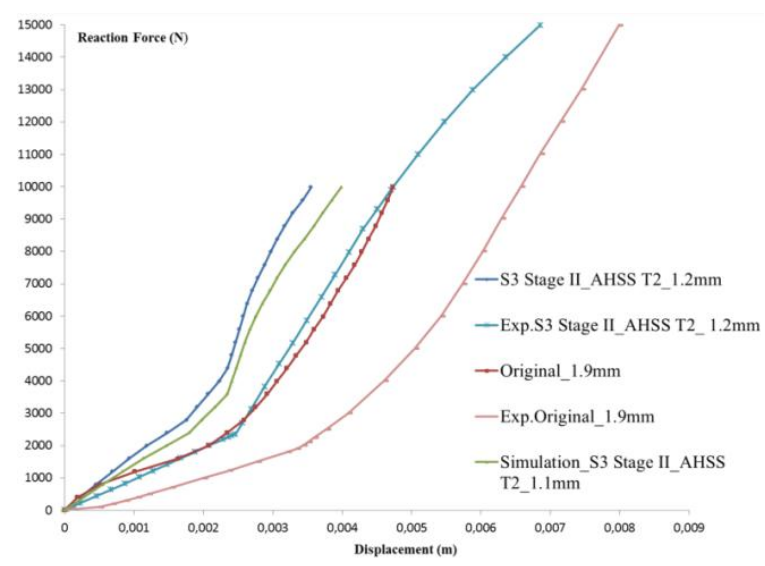

Fig. 12. Comparative analysis of the numerical and experimental tests (Exp.) for S3 Stage II AHSS T2 $1.2 \mathrm{~mm}$ and the original steel toe cap $1.9 \mathrm{~mm}$. A numerical simulated of thickness loss for the first is attempted.

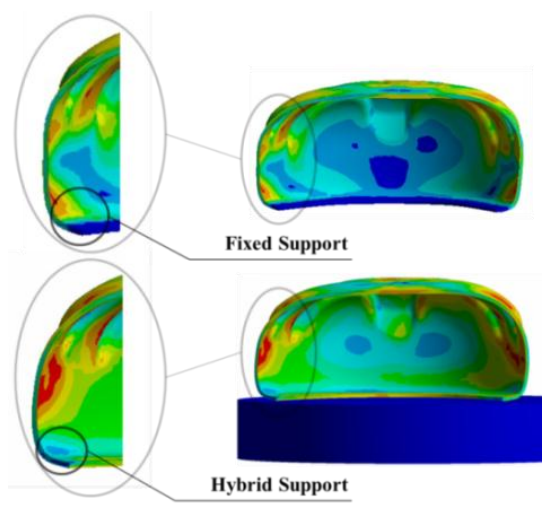

Fig. 13. FEA of the deformation behaviour using the S3 Final AHSS T2 1.2 mm model with different support approach.

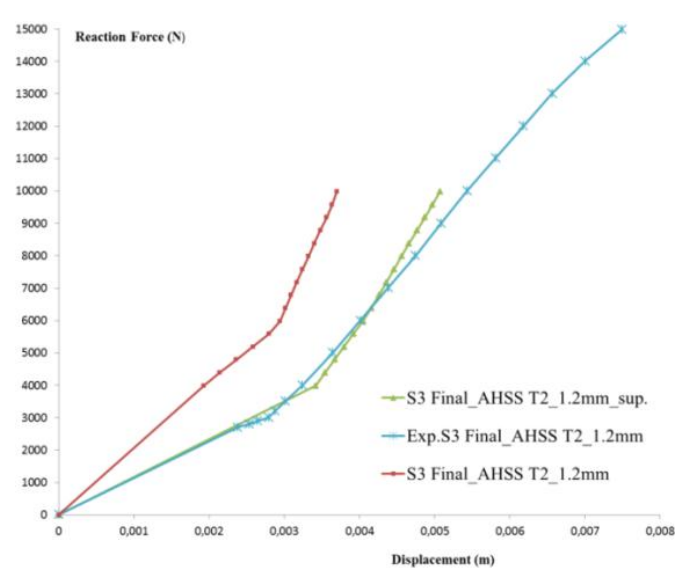

Fig. 14. Comparative analysis of numerical results for both support conditions with experimental test curve using S3 Stage II AHSS T2 $1.2 \mathrm{~mm}$.

The numerical results shown in Fig. 14 denounce a greater convergence with experimental results.

The representative numerical curve for new support conditions (represented by_sup. in Fig. 14) is way closer to the experimental test curve (exp.) than the numerical 
simulation considering ideal normative conditions. In quantitative terms, the accuracy of the quasi-static numerical simulation was substantially improved.

\section{CONCLUSIONS}

In the normative context of the quasi-static compression test, the toe cap deformation behavior was investigated through a nonlinear Finite Element Analysis. From the Project S3 - Safety Slim Shoe, the two ultimate toe cap models were selected to address the contribution of geometrical changes to potentiate AHSS applications into substantial thickness reduction. The following conclusions could be drawn:

- The study of the elasto-plastic deformation behavior has revealed relevant for the sensitivity of the mechanical properties influence in toe cap design, which aimed to achieve a substantial reduction of weight. These parameters revealed more influence than the selection of AHSS.

- Several rates of plastic deformation in compressive load conditions brought problems to convergence criterions in numerical simulation. FEA became impossible to perform load values of over $10 \mathrm{kN}$.

- Although the numerical results have revealed some quantitative clearance in the quasi-static compression program, compared to the experimental results, qualitatively the Finite Element Analysis provided a satisfactory prediction and evaluation of the toe cap performance. The accuracy of numerical results was improved considering influent parameters in study. Support conditions had shown determinant for the deformation mode definition.

- Several plastic deformations brought hardest challenges in thickness reduction of the normative components. Nevertheless, the increased focus on design in order to realize the full potential of AHSS has provided an excellent way to reduce weight and improve performance to the brand new S3 toe cap model. Higher work-hardening effects improved by local geometrical changes fulfil stiffness requirements substantially.

- The Higher thickness optimization was provided by the S3 Final Docol AHSS T3 1.1mm thickness, overcoming test results of the standard Original steel toe cap with a thickness value of $1.9 \mathrm{~mm}$. Therefore, the study presents a final scientifically validated solution with a weight saving of over $40 \%$.

\section{ACKNOWLEDGMENT}

The support from the S3-Safety Slim Shoe, FCOMP-01-0202-FEDER-018458 Project and ICC, SA Lavoro are acknowledged. The authors would like to thank SSAB Swedish Steel for the supply of experimental steel sheets, technical information and monitoring. This work was supported in part by the Project S3-Safety Slim Shoe, FCOMP-01-0202-FEDER-018458 and ICC, S.A. - Lavoro Engineering Life Wear.

\section{REFERENCES}

[1] CEN, Foot and Leg Protectors - Requirements and Test Methods for Toecaps and Penetration Resistant Inserts, 2010.

[2] A. B. Allard, "U.S. Patent No. 2, 079, 237," Washington, DC: U.S. Patent and Trademark Office, 1937

[3] B. E. Gray, "U.S. Patent No. 3, 950, 865," Washington, DC: U.S. Patent and Trademark Office, 1976.

[4] J. L. Dykeman, "U.S. Patent No. 4, 735, 003," Washington, DC: U.S. Patent and Trademark Office, 1988.

[5] L. B. Siskind, W. A. Samaha, and T. S. Warshaw, "U.S. Patent No. 4, 862, 606," Washington, DC: U.S. Patent and Trademark Office, 1989.

[6] J. L. Williams, “U.S. Patent No. 6, 367, 170," Washington, DC: U.S. Patent and Trademark Office, 2002.

[7] C. Frulla, "U.S. Patent No. 7, 062, 868," Washington, DC: U.S. Patent and Trademark Office, 2006.

[8] Y. Tanaka, M. Hirota, and T. Ishida, "U.S. Patent No. 6, 907, 681," Washington, DC: U.S. Patent and Trademark Office, 2005.

[9] R. Almaguer, F. C. Ruiz, C. A. F. Aragon, R. Z. Guzman, R. A. A. Medina, and C. V. Guerrero, "European Patent No. EP 2223621," Munich, Germany: European Patent Office, 2010.

[10] L. J. Deka, S. D. Bartus, and U. K. Vaidya, "Multi-site impact response of S2-glass/epoxy composite laminates," Composites Science and Technology, vol. 69, no. 6, pp. 725-735, 2009.

[11] M. P. Ho and K. T. Lau, "Design of an impact resistant glass fibre/epoxy composites using short silk fibres," Materials and Design, vol. 35, pp. 664-669, 2012

[12] S. Lee, T. Lim, and D. Lee, "Damage tolerance of composite toe cap," Composite Structures, vol. 67, no. 2, pp. 167-174, 2005.

[13] M. Kuhn, J. Nowacki, and N. Himmel, "Development of an innovative high performance FRP protective toe cap," Journal of Materials: Design and Applications, vol. 219, no. 2, pp. 91-109, 2005.

[14] SSAB, Sheet Steel Forming Handbook-Plastic Deformation, $3^{\text {rd }}$ ed. 1996.

[15] SSAB, AHSS for the Automotive Industry, $2^{\text {nd }}$ ed. 2012.

[16] W. Bernert, M. Bzdok, and J. Davis, “Advanced high-strength steel product and process applications guidelines," Auto/Steel Partnership, 2008.

[17] R. Kuziak, R. Kawalla, and S. Waengler, "Advanced high strength steels for automotive industry," Archives of Civil and Mechanical Engineering, vol. 8, no. 2, pp. 103-117, 2008.

[18] L. S. Costa, V. J. Silva, H. Puga, N. Peixinho, and J. P. Mendonça, "Sustainable reverse engineering methodology assisting $3 \mathrm{~d}$ modeling of footwear safety metallic components, presented at the International Mechanical Engineering Congress \& Exposition - ASME2013, San Diego, USA, 2013.

[19] H. H. Lee, "Finite element simulations with ANSYS workbench 12 theory - applications - case studies," 2010.

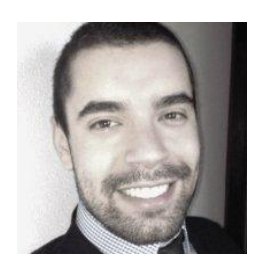

Sérgio L. Costa was born in Vila Nova de Famalicão, Portugal in 1985. In December of 2009 , Sérgio L. Costa received the M.Sc. in mechanical engineering from the University of Minho. Since 2012. He is a Ph.D. candidate at University of Minho and researcher at Centre for Mechanical and Materials Technologies - CT2M. Professor Nuno Peixinho and Professor João P. Mendonça are his $\mathrm{Ph} . \mathrm{D}$. supervisors and the work is developed in industrial context of the Project S3-Safety Slim Shoe with ICC, S.A. - Lavoro Group and SSAB, Swedish Steel AB. His research is focused in numerical simulation, AHSS application, optimization of energy absorption capacity, quasi-static compression, structural impact and dynamic material behavior. 